Special Issue Review

\title{
Recent Progress in Pulse Power Technology and its Applications
}

\author{
Kazuhiko Horioka* Member \\ Weihua Jiang** Member
}

\begin{abstract}
Pulse power has expanded its regime in technological and application fields. The state-of-art of switching technology shifts the trend of pulse power from huge and single shot systems to compact and highly repetitive generators. This extends the pulse power application into industrial, environmental, biological, medical, and also scientific fields. The progress has enabled us to have high energy density plasma and/or well-defined extreme conditions with low cost and good reproducibility. Plasmas produced by compact pulse power devices are proposed as a new tool in small laboratories for high energy density physics and its applications.
\end{abstract}

Keywords : pulsed power, plasma, high voltage, pulsed discharge, power semiconductor device, high energy density

\section{Introduction}

Pulsed power has been studied for decades as one of the key technologies for developments in nuclear fusion, high-power lasers, accelerators, and high energy-density physics. Recently, its applications have expanded into many new areas such as environmental, biological and medical fields ${ }^{(1)-(6)}$.

At the same as the new applications emerge, pulsed power itself has undergone a transition from large and single-shot (or low repetition rate) systems to small and highly repetitive generators. This transition has enabled pulsed power to become application-oriented and commercially available.

In recent years, two major trends are clearly noticeable in the development of pulsed power technology ${ }^{(7)}$. One is the fast spread of the power semiconductor devices. Semiconductor switching devices such as metal-oxide-semiconductor field-effect-transistor (MOSFET) and insulated-gate bipolar transistor (IGBT) have been widely used in many pulsed power generators and pulsed high-voltage modulators for applications in high-energy accelerators, excimer lasers, and atmospheric discharges for gas-treatment and sterilization.

The other trend is in the development of inductive energy storage generator scheme. With inductive energy storage, the energy is released to the load when the switch parallel to the load is opened. In an L-R circuit, the pulse duration is proportional to $\mathrm{L} / \mathrm{R}$ and, therefore, it is relatively easy to generate very short high-voltage pulse for a load of high impedance. In addition, the output voltage is determined by the current gradient $(\mathrm{dI} / \mathrm{dt})$ in the inductor, which can be very high if the switch opens fast.

By a properly formed electromagnetic pulse, we can make an extremely high energy density condition that was utilized for generation of high power lasers, high current particle beams, and thermonuclear fusion. During the last two decades, power semiconductors extend the capability of pulse power devices and they have found various new fields such as materials, energy, environmental, biochemical, medical applications and also intense

* Department of Energy Sciences, Tokyo Institute of Technology 4259 Nagatsuta, Yokohama 226-8502

** Extreme Energy-Density Research Institute, Nagaoka University of Technology

1603-1, Kamitomioka, Nagaoka 940-2188 radiation sources for lithography. When a conditioned electromagnetic energy is put into a certain material, they can be heated up to form a plasma with a condition appropriate for coherent or incoherent emission of extreme ultraviolet (EUV) radiation. The source plasma has advantages for various applications, such as EUV microscopy, spectroscopy, materials processing, and micro-lithographic imaging. In particular, a bright radiation from a discharge produced high energy density plasma is expected to be a possible light source in EUV region for the next generation lithography.

Recently, plasmas produced by compact (laboratory scale) pulse power devices are proposed as new tools for high energy density (HED) physics and also for material science using the extreme condition. The use of pulsed high electric field with durations in nanosecond range has been proposed for biological and medical applications.

In this paper, we give a brief review on recent development on semiconductor based pulsed power generator and that using inductive energy storage, together with their recent applications.

\section{Semiconductor-Based Repetitive Generators}

Besides MOSFET and IGBT which are already widely used in pulsed power systems, other power semiconductor devices are also being studied for future pulsed-power applications. Among them, silicon carbide FET and static induction thyristor are promising candidates.

Silicon carbide ( $\mathrm{SiC})$, due to its wider band-gap compared with that of silicon, is expected to make a superb material for power semiconductor devices. In recent years, the progress in device manufacturing has successfully led to the commercial availability of $\mathrm{SiC}$ power devices.

We have tested a silicon carbide junction FET (SiC-JFET) for its switching speed and switching loss, in order to evaluate its applicability to high-voltage modulators. The device we have tested is made by SiCED Electronics Development GmbH \& Co. $\mathrm{KG}$, the photo of which is shown in Fig. 1.

The experiments were carried out with switching voltage of $1 \mathrm{kV}$. Figure 2 shows the waveform of the device voltage obtained at temperature of $20{ }^{\circ} \mathrm{C}$ and $200^{\circ} \mathrm{C}$. From Fig. 2 the turn-on and turn-off times were obtained to be $\sim 20 \mathrm{~ns}$ and $\sim 38 \mathrm{~ns}$ 


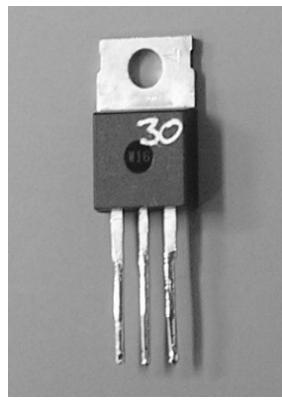

Fig. 1. Photograph of SiC-JFET tested for pulsed power switching

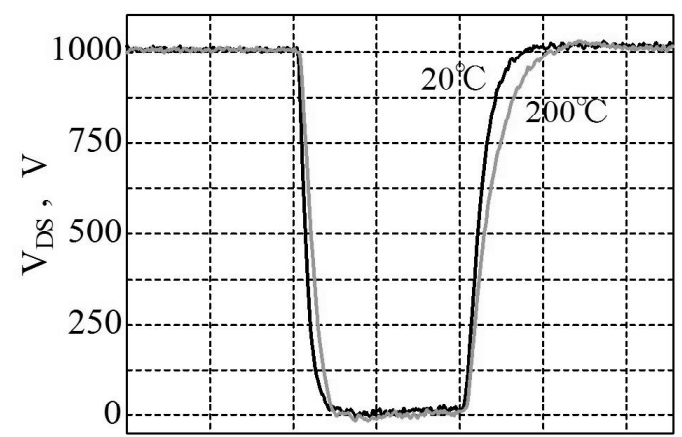

Time , $100 \mathrm{~ns} / \mathrm{div}$

Fig. 2. Waveforms of the voltage obtained on SiC-JFET, for temperature of $20^{\circ} \mathrm{C}$ and $200{ }^{\circ} \mathrm{C}$, respectively

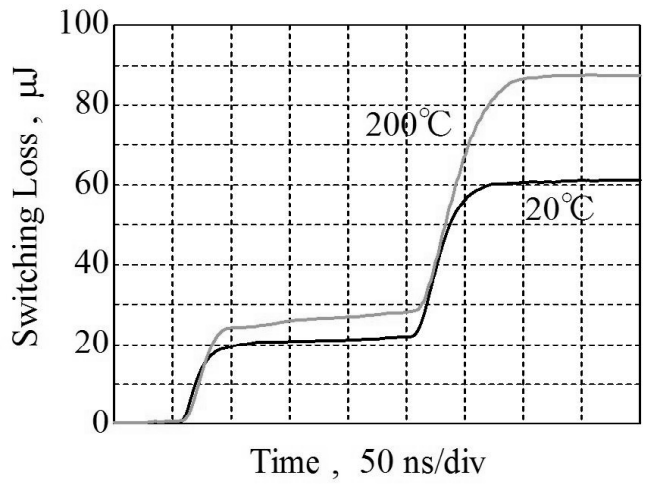

Fig. 3. Switching loss calculated from the voltage and current waveforms

(for $20^{\circ} \mathrm{C}$ ) and $\sim 28 \mathrm{~ns}$ and $\sim 60 \mathrm{~ns}$ (for $200{ }^{\circ} \mathrm{C}$ ), respectively.

The on-time resistance of the SiC-JFET was obtained to be $0.3 \Omega$ (for $20{ }^{\circ} \mathrm{C}$ ) and $\sim 1 \Omega$ (for $200{ }^{\circ} \mathrm{C}$ ) respectively. The switching loss has been calculated by integrating the product of the device voltage and circuit current. Figure 3 shows the result giving the values of $\sim 60 \mu \mathrm{J} /$ pulse for $20{ }^{\circ} \mathrm{C}$ and $\sim 85 \mu \mathrm{J} /$ pulse for $200^{\circ} \mathrm{C}$.

The above results have shown that, both in switching time and in switching loss, the SiC-JFET has similar performance with silicon-based MOSFET. However, the capability of high-temperature operation is clearly an advantage. In addition, since $\mathrm{SiC}$ devices are under rapid development, we can expect more superb devices in the following years.

Another type of semiconductor switch is the static-induction thyristor (SIThy) which was developed by NGK Insulators, Ltd.

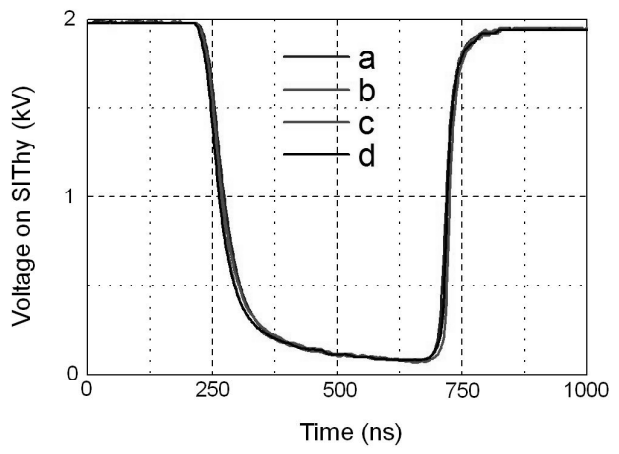

Fig. 4. Waveforms of SIThy voltage obtained for four devices that are used to form a full-bridge generator shown in Fig. 5

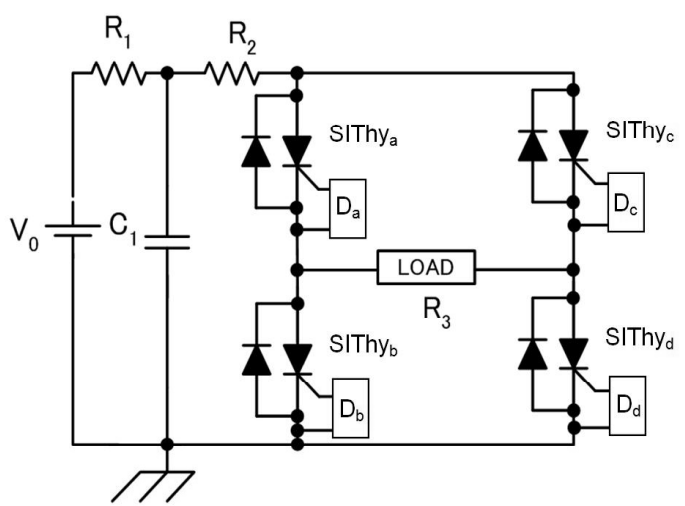

Fig. 5. Circuit of full-bridge generator using four identical SIThys

and Shindengen Electric Manufacturing, Ltd. The study of SIThy is an attempt to evaluate its characteristics for application to induction synchrotron ${ }^{(8)}$.

The present induction synchrotron is powered by MOSFET modulators that use multiple MOSFETs in order to guarantee the switching voltage $\mathrm{e}^{(9)}$. However, the large number of switching elements and their supporting components, including the driver circuits and the power supplies, results in large volume and complicated system. Therefore, there is a strong desire to replace the multiple MOSFETs by a single device that has higher voltage capability.

SIThy is studied as a potential candidate for replacing the multiple MOSFETs. It has much higher power capacity than any kind of MOSFET ${ }^{(10)}$. However, its switching speed is an unclear issue for repetition in $\mathrm{MHz}$ range. For this reason, our interest in the initial experiment is at the MHz operation of the SIThy.

The switching performance of single SIThy devices are shown in Fig. 4, from which we have obtained the turn-on and turn-off times of $\sim 100 \mathrm{~ns}$ and $\sim 40 \mathrm{~ns}$, respectively. In addition, from Fig. 4 , we see that the on-time resistance of SIThy is relatively high. The switching loss calculated from the voltage and current waveforms gives a result of $\sim 2 \mathrm{~mJ} /$ pulse.

Figure 5 shows the circuit of a full-bridge generator using four SIThys in order to generate bipolar output voltage for induction synchrotron. The four devices have been confirmed to perform identically as shown in Fig. 4. They are driven by four identical driver circuits (marked by $D_{a} \sim D_{d}$ in Fig. 5) that are optically controlled. The voltage waveform obtained on the resistive load is shown in Fig. 6 . The bipolar output voltage of $\pm 1.2 \mathrm{kV}$ has 


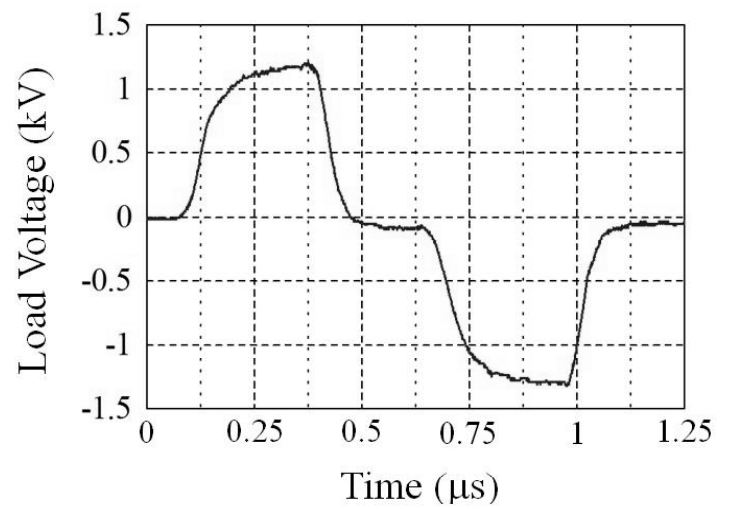

Fig. 6. Voltage waveform obtained on the load resistor, by the circuit shown in Fig. 5

been obtained, although the switching time and the voltage flatness need to be improved.

\section{Inductive Energy Storage with Opening Switch}

Pulsed power generator using inductive energy storage is known to be suitable to relatively high-impedance load. For this reason, it is widely studied recently for applications to atmospheric discharges of very short pulse length.

Inductive energy storage scheme needs an opening switch with capabilities in fast opening and high hold-off voltage. Among many kinds of opening switch, the semiconductor opening switch (SOS) developed in Russia has attracted considerable attentions $^{(10)(11)}$.

Typical SOS-based generator is shown in Fig. 7. The IGBT and pulse transformer (PT) allows voltage multiplication during energy transfer between capacitors $\mathrm{C}_{0}$ and $\mathrm{C}_{1}$. When the charging of $\mathrm{C}_{1}$ finishes, magnetic switch $\mathrm{MS}_{1}$ saturates allowing energy transfer to $\mathrm{C}_{2}$, while pumping SOS with a forward current. At the maximum $\mathrm{C}_{2}$ voltage, magnetic switch $\mathrm{MS}_{2}$ saturates, which initiates a pulsed high current in the reverse direction of the SOS diodes. The sudden cutoff of the SOS reverse current induces a pulsed high voltage to the load.

Figure 8 shows the waveforms obtained by a generator using circuit design of Fig. 7. Here, the load is a discharge gap in air with electrode distance of $1 \mathrm{~cm}$. The rise-time is $\sim 20 \mathrm{~ns}$ and the peak voltage is limited by the breakdown between the gap. The pulse length is short enough to avoid transition from streamer discharge to an arc. This generator can operate at repetition rate of $1 \mathrm{kHz}$.

Although the physical principle of the SOS is very simple, the practical driving circuit is relatively complicated which usually require magnetic switches, as in the circuit of Fig. 7. An attempt has been made to get rid of the magnetic switch and drive the SOS generator using a full-semiconductor circuit. Figure 9 shows a circuit designed for this purpose. In this circuit, $\mathrm{C}_{1}$ and $\mathrm{FET}_{1}$ are for driving the SOS forward current and $\mathrm{C}_{2}$ and $\mathrm{FET}_{2}$ are for driving the SOS reversed current. The delay time between them is properly controlled by an external driving circuit. In Fig. 9, $\mathrm{C}_{3}$ and $\mathrm{FET}_{3}$ are for pulsed charging $\mathrm{C}_{2}$.

Without magnetic switch, the time required for magnetic flux restoring is no longer necessary. Therefore, in principle, the generator can be operated at very high repetition rate. A

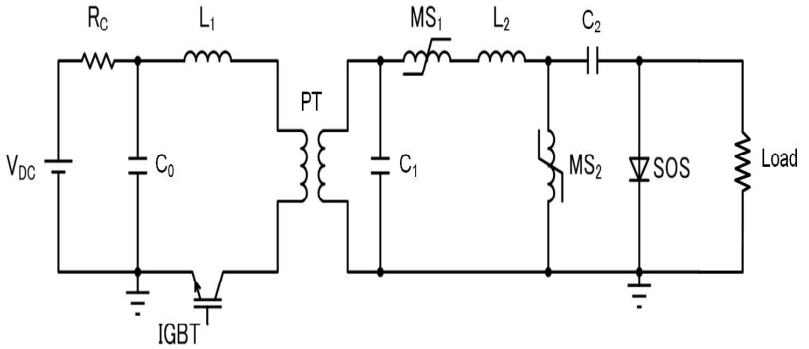

Fig. 7. Typical pulsed power generator circuit using inductive energy storage with SOS as opening switch

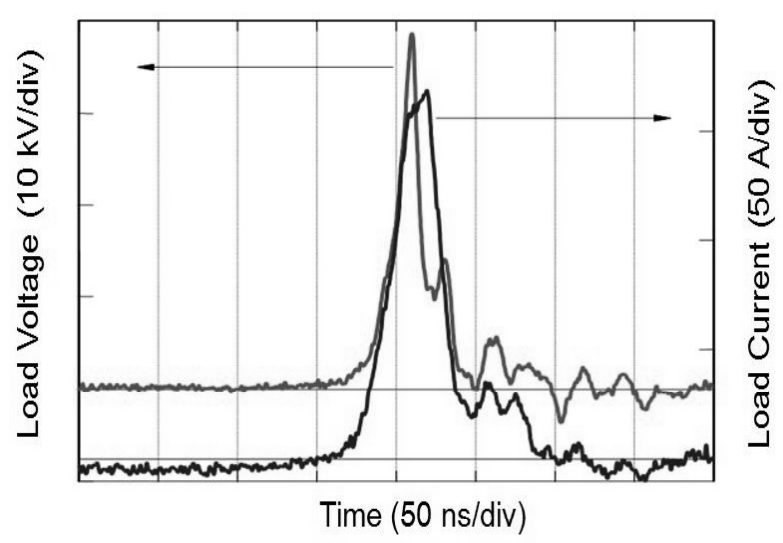

Fig. 8. Waveforms of output voltage and current obtained from the circuit shown in Fig. 7

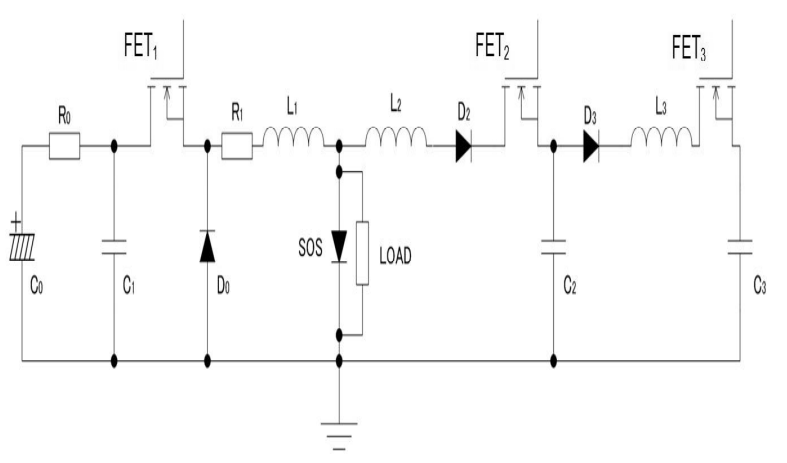

Fig. 9. Pulsed power generator where SOS is driven by a full-semiconductor circuit

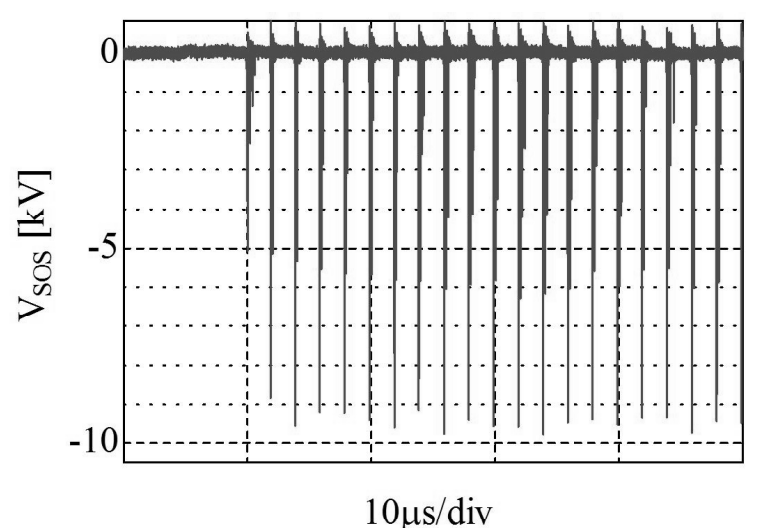

Fig. 10. Voltage waveform obtained on the $1-\mathrm{k} \Omega$ load using the generator shown in Fig. 9 
demonstration experiment was carried out at repetition rate of 500 $\mathrm{kHz}$ (in burst mode) and the result is shown in Fig. 10. The experimental results have shown that the output voltage on the resistive load of $1 \mathrm{k} \Omega$ has pulse width of $\sim 30 \mathrm{~ns}$ and peak value of $\sim 9 \mathrm{kV}$. This experiment is the first attempt to drive the SOS with power semiconductor device and it has obtained the highest repetition rate of SOS-based generator.

\section{Plasma-Based Bright Radiation Sources}

The source plasma for short wavelength radiation can be produced through a pinching process driven by pulse power generator. A schematic of the pinching plasma is shown in Fig.11. As shown in the figure, self magnetic field $B_{\theta}=\left(\mu_{0} I^{2}\right) /\left(8 \pi R^{2}\right)$ induced by the plasma current $I$, confines the high-temperature, radiating plasma. Thus the plasma parameters appropriate for the radiating plasma, both incoherent and coherent extreme ultraviolet sources, can be basically controlled by the discharge condition ${ }^{(12)}$.

4.1 Coherent Radiation Sources When parameters of a highly ionized plasma is properly controlled, the plasma can make a lasing condition. In the discharge laser scheme, a fast pulse power drives the plasma in a capillary tube to make a highly ionized, appropriate plasma condition for stimulated amplification radiation in $\mathrm{Ne}$ (neon)-like or $\mathrm{H}$ (hydrogen)-like ions. For example, a typical operating condition of the discharge pumped Ne-like $\mathrm{Ar}$ laser is shown in Table.1.

In the discharge pumped laser scheme, a fast pulse power generator drives a discharge plasma in a capillary tube to make a long, cylindrical lasing plasma. Continuous efforts are made for energetic operation at shorter wavelength. Correlation between the discharge dynamics and the plasma parameter that leads to a lasing condition are investigated and the dominant operating parameters for an energetic lasing are clarified, using experimental, analytical, and numerical approaches ${ }^{(13)(14)}$. The results indicate that the dynamics of pinching plasma plays a crucial role to make appropriate lasing plasma.

4.2 Incoherent Radiation Sources EUV lithography is considered to be the successor of optical processing ${ }^{(15)}$. In the lithography system using EUV light source, at EUV-wavelength region any materials absorb the radiation so strongly that the optics must be composed of reflecting components and the entire process must be taken place in vacuum. That requests the source should be a plasma in vacuum and the average power has to be more than $1 \mathrm{~kW}$, at wavelength of $13.5 \mathrm{~nm}$, into a bandwidth of $2 \%$. Two different approaches are developing for making the EUV source plasma; laser produced plasmas and discharge produced (pulse power) plasmas. Mainly from the point of construction cost and system efficiency, interests in discharge produced plasmas are increasing.

Generally, the discharge produced plasma is compressed and heated through a pinching process. At the stagnation phase, the compressed plasma emits the radiation. When we control the operating parameters so as to make a proper plasma parameter, the compressed plasma is expected to emit a bright radiation centered at $13.5 \mathrm{~nm}$. Compared with the laser produced plasma, the discharge plasma has a number of advantages ${ }^{(16)-(18)}$. In particular, the discharge device has a lot of control parameters, such as electrode geometry, working gas density, its distribution and current shape. Optimization of these parameters should bring a source quality sufficient for the lithography systems.

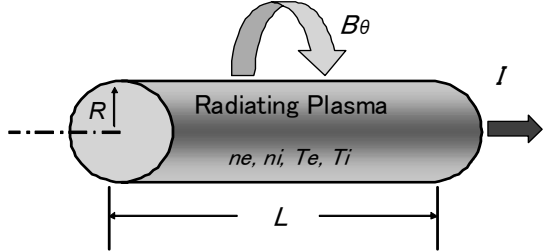

Fig. 11. Cylindrical radiating plasma formed by pinching process

Table 1. Typical operating condition of pulse-powered discharge (Ne-like argon) laser

\begin{tabular}{|l|c|}
\hline Driver Energy & $\sim 1 \mathrm{~kJ}$ \\
\hline Capillary Diameter & $3 \mathrm{~mm}$ \\
\hline Capillary Length & $100-300 \mathrm{~mm}$ \\
\hline Filling Pressure & $100-1000 \mathrm{mTorr}(\mathrm{Ar})$ \\
\hline Current Rise Rate & $10^{11}-10^{12} \mathrm{~A} / \mathrm{sec}$ \\
\hline Pre-ionization Curent & $50-90 \mathrm{~A}$ \\
\hline
\end{tabular}

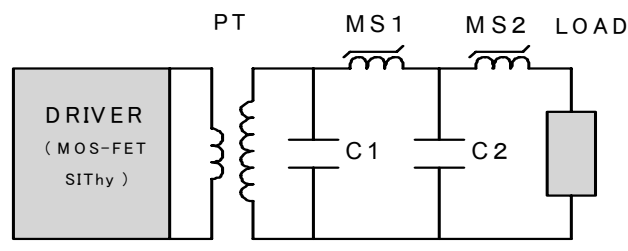

Semiconductor Based Step-up Magnetic Pulse Pulse Forming Unit Transformer Compression Unit

Fig. 12. Schematic of a pulse power generator for plasma source

\subsection{Pulse Power System for Plasma Radiation Source}

The discharge produced plasma is generated by a fast pulse power generator with high repetition capability and long life time. Switching devices such as MOS-FET(Metal-oxide semiconductor field effect transistor), SIThy(Static induction thristor), IGBT are the key components for the pulse powered generator.

A schematic of the typical pulse power device for the discharge produced radiating plasma is shown in Fig. 12. The device consists of a primary pulse forming section driven by the semiconductor power devices, a step-up pulsed transformer PT, and two-stage magnetic pulse compression unit that is driven by magnetic switches MS. Through the pulse-forming and compression processes, finally it drives a discharge current of $\sim 10 \mathrm{kA}$ with a few $100 \mathrm{nsec}$ of pulse width.

\section{Pulse Power Driven High Energy Density Plasma}

Plasmas at high density and/or high temperature state are of primary concern for optimization of the target structure of inertial confinement fusion, exploration of astrophysics, and also for material science at extreme condition ${ }^{(19)-(21)}$.

Dense cylindrical plasma can be produced using a pulse-power driven exploding wire discharge in water, in which the plasma is tamped and stabilized by the surrounding water ${ }^{(20)}$. A schematic diagram for the experiment on wire explosion is shown in Fig.13.

The structure of strong shock waves in a high- $Z$ gas is expected to be completely different from that of conventional hydrodynamic shock waves. A shock heated, high temperature plasma is produced in a compact pulse power device ${ }^{(22)}$. Results show that extremely strong shock wave is produced in the device.

Figure 13 is a density-temperature diagram, which shows a schematic of parameter ranges of high energy density plasma in 


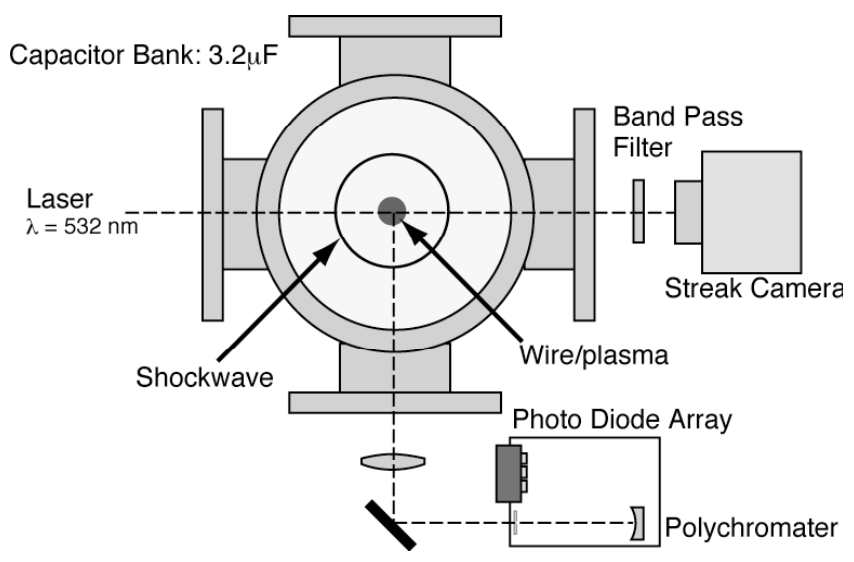

Fig. 13. Experimental set-up for dense plasma formation using wire explosion in water

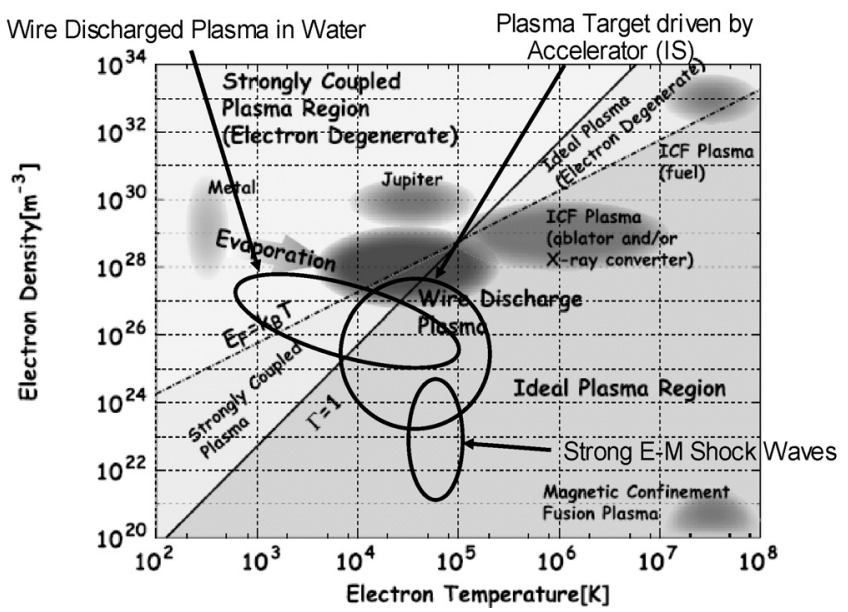

Fig. 14. Expected parameter ranges of pulse power driven high-energy-density plasma

our world and also the expected parameter values driven by the compact pulse power devices. Advantages of the pulse power driven HED plasma are compactness of the device, capability of making larger spacial and temporal scale, and also providing a well-defined condition.

\section{Biological and Medical Applications}

Concerns have been raised regarding the relationship between pulsed electric field and biological effects. The effect of pulsed electric fields on bacteria and aquatic nuisance species was explored and pulse width dependence of electrical energy required for lysing of bacteria, stunning of aquatic species was investigated. The pulse duration needs to be short to prevent thermal effects. Nano-second electric fields have the following advantages: (i) Negligible thermal heating, (ii) the ability to develop large electric field with a lower energy input, (iii) the capacity to create large trans-membrane potentials across sub-cellular structure. Applications of the pulsed electric field technique are in biofouling prevention, debacterialization, and in the field of medicine, e.g., for delivery of chemotherapeutic drugs into tumor cells $^{(23)(24)}$.

Based on capacitive coupling to cell substructures, this has a potential to affect transport process across subcellular membranes. Other studies also indicates that it triggers intracellular processes such as programmed death that can be used for cancer treatment. On the other hand, researches on the biological effects of electromagnetic field have been conducted for more than twenty years from the point view of human health ${ }^{(25)}$. It will be very important to conduct comparative studies on the biological effects of pulsed electric field.

\section{Conclusions and Prospect}

Supported by the technological progress of semiconductor power devices, pulse power technology is expanding its territory into new fields; industrial, environmental, biological fields, and also basic science. Highly repetitive and high average-power devices have been developed and they are expected to be useful in the variety of applications. Among them, the EUV lithography is probably the most challenging application. Although, a lot of technological and plasma physical issue must be overcome, the basic behavior of plasma radiation source can be predictable and scalable with the aid of numerical analysis based on Magneto-hydrodynamics. All of the experimental, analytical, and numerical results indicate advantageous property of the discharge produced radiating plasma as the high average power bright radiation source, in particular, for the next generation lithography light source.

Plasmas produced using compact pulse power devices are proposed for HED physics and its applications. Results show that plasmas made by wire explosion in water can be appropriate sources for study on the equation of state and transport coefficients in a wide range of parameters. Shock heated plasmas produced by pulse-powered electro-magnetic force in a pair of conical electrodes, are demonstrated to be a suitable one-dimensional test sample for high temperature hydrodynamics involving ionization relaxation and a radiative energy transfer. Advantages of pulse-powered approaches to these fields are compactness, cost effectiveness, and also ability of providing a well-defined condition with large scale-length.

Application of pulsed high electric field to biological and/or medical field is considered to be still in an infant stage. Much more efforts should be needed for evaluating the usefulness in the practical biological field.

Although there are a lot of issues, new technologies are expected to be arisen from the newly developed pulse-power tools.

(Manuscript received Aug. 16, 2007)

\section{References}

(1) S. Daito, F. Tochikubo, and T. Watanabe : "Improvement of NOx Removal Efficiency Assisted by Aqueous-Phase Reaction in Corona Discharge", Jpn, J. Appl. Phys., Vol.39, No.8, pp.4914-4919 (2000)

(2) W. J. M. Samaranayake, Y. Miyahara, T. Namihira, S. Katsuki, R. Hackam, and H. Akiyama : "Ozone Generation in Dry Air Using Pulsed Discharge With and Without a Solid Dielectric Layer", IEEE Trans. Dielect. Elect. Insulation, Vol.8, No.4, pp.687-697 (2002)

(3) B. R. Locke, A. Ichihashi, H. H. Kim, and A. Mizuno : "Diesel Engine Exhaust Treatment with a Pulsed Streamer Corona Reactor Equipped with Reticulated Vitreous Carbon Electrodes", IEEE Trans. Industr. Applic., Vol.37, No.3, pp.715-723 (2001)

(4) H-K. Lee, J. Suehiro, M. Hara, D-C. Lee, and M-H. So : "Energy Efficiency Improvement of Electrical Sterilization Using Oscillatory Waveforms from a RLC Discharging Circuit", IEEE Trans. Dielect. Elect. Insulation, Vol.7, No.6, pp.872-874 (2000)

(5) K. Takaki, H. Kirihara, C. Noda, S. Mukaigawa, and T. Fujiwara : "Production of pulse glow discharge in atmospheric pressure nitrogen using needle-array electrode", Jpn. J. Appl. Phys., Vol.45, pp.8241-8245 (2006) 
(6) T. Namihira, D. Wang, S. Katsuki, R. Hackam, and H. Akiyama : "Propagation Velocity of Pulsed Streamer Discharges in Atmospheric Air", IEEE Trans. Plasma Sci., Vol.31, No.5, pp.1091-1094 (2003)

(7) W. Jiang, K. Yatsui, K. Takayama, M. Akemoto, E. Nakamura, N. Shimizu, A. Tokuchi, S. Rukin,V. Tarasenko, and A. Panchenko : "Compact Solid-State Switched Pulsed Power and Its Applications", Proc. IEEE, Vol.92, issue 7, pp.1180-1196 (2004)

(8) K. Takayama and J. Kishiro : "Induction Synchrotron", Nucl. Instrum. Methods Phys. Res. A, Accel. Specrom. Detect. Assoc. Equip., Vol.A451, pp.304-317 (2000)

(9) K. Takayama, K. Koseki, K. Torikai, A. Tokuchi, E. Nakamura, Y. Arakida, Y. Shimosaki, M. Wake, T. Kouno, K. Horioka, S. Igarashi, T. Iwashita, A. Kawasaki, J. Kishiro, M. Sakuda, H. Sato, M. Shiho, M. Shirakata, T. Sueno, T. Toyama, M. Watanabe, and I. Yamane : "Observation of the Acceleration of a Single Bunch by Using the Induction Device in the KEK Proton Synchrotron", Phys. Rev. Lett., Vol.94, p.144801 (2005)

(10) R. Hironaka, E. Hotta, A. Okino, M. Maeyama, K .C. Ko, and N. Shimizu : "Performance of pulsed power generator using high-voltage static induction thyristor", IEEE Trans. Plasma Sci., Vol.28, pp.1524-1527 (2000)

(11) A. I. Bushlyakov, et.al. : "Solid-state SOS-based generator providing a peak power of 4GW", IEEE Trans. Plasma Sci., Vol.34, pp.1873-1878 (2006)

(12) K. Horioka, E. Hotta, M. Shiho, et.al. : "Coherent or Incoherent Radiation Soource in XUV region using Fast Pulse-power Devices", Proceedngs of the $5^{\text {th }}$ International Symposium on Pulsed Power and Plasma Applications, pp.57-60, Korea (2004)

(13) G. Niimi, Y. Hayashi, N. Sakamoto, M. Nakajima, A. Okino, M. Watanabe, K. Horioka, and E. Hotta : "Development and characterization of a low current capillary discharge for X-ray laser studies", IEEE Trans. Plasma Sci., Vol.30, pp.616-621 (2002)

(14) M. Masnavi, M. Nakajima, and K. Horioka : "Numerical Study of a Capillary Discharged Neon-Like Argon X-ray Laser", IEEJ Trans. FM, Vol.126, No.4, pp.250-255 (2006)

(15) W. T. Shilfvast : "Intense EUV Incoherent Plasma Sources for EUV Lithography and Other Applications", IEEE J. Quantum Electron, Vol.35, pp.700-708 (1999)

(16) M. Masnavi, M. Nakjima, A. Sasaki, E. Hotta, and K. Horioka : "Approach to optimize conversion efficiency of discharge-pumped plasma extreme ultraviolet sources", Appl. Phys. Lett., Vol.87, p.111502 (2005)

(17) M. Masnavi, M. Nakajima, A. Sasaki, E. Hotta, and K. Horioka : "Potential of discharge-pumped lithium plasma as an extreme ultraviolet source", Appl. Phys. Lett., Vol.89, p.031503 (2006)

(18) S. R. Mohanty, T. Sakamoto, Y. Kobayashi, et.al. : "Influence of electrode separation and gas curtain on extreme ultraviolet emission of a gas jet z-pinch source", Appl. Phys. Lett., Vol.89, p.041502 (2006)

(19) R. Davidson, Ed. : Frontiers in High Energy Density Physics-The X-Games of Contemporary Science, National Academic Press (2003)

(20) R. P. Drake : High-Energy-Density Physics, Springer (2006)
(21) K. Horioka, T. Kawamura, M. Nakajima, et.al. : "High-energy-density physics researches based on Heavy Ion Beam and Pulse Power Devices", Nuclear Instr. Meth., Vol.A-577, pp.298-302 (2007)

(22) T. Sasaki, Y. Yano, M. Nakajima, T. Kawamura, and K. Horioka : "Warm-Dense-Matter Studies using Pulse-Powered Wire Discharges in Water", Laser \& Particle Beams, Vol.24, pp.371-380 (2006)

(23) K. H. Schoenbach, F. E. Peterkin, R. W. Alden, and S. J. Beebe : "The effect of electric field on biological cells: Experiments and applications", IEEE Trans. Plasma Sci., Vol.25, pp.284-292 (1997)

(24) K. H. Schoenbach, S. Katsuki, R. H. Stark, E. S. Buescher, and S. J. Beebe : "Bioelectric-New applications for pulsed power technology", IEEE Trans. Plasma Sci., Vol.30, pp.293-300 (2002)

(25) T. Shigemitsu, K. Yamazaki, S. Nakasono, and M. Kakikawa : "A Review of Studies of the Biological Effcets of Electromagnetic Fields in the Intermediate Frequency Range", IEEJ Trans., Vol.2, Issue 4, pp.405-412 (2007)

Kazuhiko Horioka

(Member) was born on Oct.5, 1951, in Osaka. He received B.E. M.E. and $\mathrm{PhD}$ degrees from Osaka University, in 1974, 1976 and 1980, respectively. He is a professor in Department of Energy Sciences, Interdisciplinary Graduate School of Science and Engineering, Tokyo Institute of Technology and is currently serving as the chairperson of the Technical Committee on Pulsed Electromagnetic Energy. His interests include the physics and applications of pulsed electromagnetic energy, high energy density plasmas, and high current particle beams.
Weihua Jiang

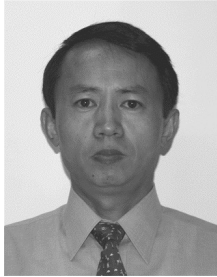

(Member) was born in Beijing, China in 1962. He received the B.Sc. degree in engineering physics from the National University of Defense Technology, Changsha, China, in 1982, and the M.S. degree in plasma physics from the Institute of Atomic Energy, Beijing, China, in 1985. He received the Ph.D. degree in electrical engineering from Nagaoka University of Technology, Nagaoka, Japan, in 1991. He is currently a Professor at Extreme Energy-Density Research Institute of Nagaoka University of Technology. His research interests include pulsed power technology and high-power microwave generation. 